\title{
INFILTRACIÓN BILATERAL DEL NERVIO ÓPTICO EN UN CASO DE LEUCEMIA AGUDA LINFOBLÁSTICA DE CÉLULAS T EN REMISIÓN
}

\author{
BILATERAL OPTIC NERVE INFILTRATION IN ACUTE \\ LYMPHOBLASTIC LEUKEMIA IN REMISSION
}

\author{
MATEO J ${ }^{1}$, ABARZUZA R ${ }^{1}$, NÚÑEZ E ${ }^{1}$, CRISTÓBAL JA ${ }^{2}$
}

\section{RESUMEN}

Caso clínico: Varón de 18 años diagnosticado de leucemia aguda linfoblástica de células T (LAL-T), que estando en remisión completa tras recibir quimioterapia, presentó infiltración bilateral del nervio óptico.

Discusión: La infiltración del nervio óptico puede aparecer como un signo aislado de recaída extramedular del LAL-T que preceda en meses a la recaída hematológica.

Palabras clave: Infiltración leucémica, nervio óptico, leucemia aguda linfoblástica, inducción, remisión, recaída.

\section{ABSTRACT}

Case report: An 18-year-old male affected by acute lymphoblastic leukemia (ALL) after having reached complete remission after chemotherapy developed bilateral optic nerve infiltration.

Discussion: Infiltration of the optic nerve may appear as an isolated sign of extramedullary relapse of ALL months in advance of the hematologic relapse (Arch Soc Esp Oftalmol 2007; 82: 167170).

Key words: Leukemic infiltration, optic nerve, leukemia, lymphocytic, acute, remission induction, recurrence.

\section{INTRODUCCIÓN}

La leucemia aguda linfoblástica de células $\mathrm{T}$ (LAL-T) es una patología que aparece principalmente en niños y adolescentes, sobre todo varones. En la presentación suelen aparecer astenia, anorexia, y pérdida de peso, debidas a la insuficiencia medular y la infiltración de órganos (es típica la presencia de masa mediastínica y no es rara la afec- tación del sistema nervioso central en el momento del diagnóstico) (1).

La clínica ocular en estos pacientes varía mucho (conjuntivitis, hifema, proptosis, hemorragias retinianas, desprendimiento de retina...) (2) y se ve cada vez con más frecuencia, debido al aumento de la esperanza de vida gracias a la utilización de agentes quimioterápicos cada vez más efectivos (3). Dicha clínica puede deberse tanto a la enfermedad

\footnotetext{
Recibido: 31/3/06. Aceptado: 16/2/07.

Hospital Clínico Universitario Lozano Blesa. Zaragoza. España.

1 Licenciado en Medicina.

2 Doctor en Medicina.

Correspondencia:

Javier Mateo Gabás

Hospital Clínico Universitario «Lozano Blesa»

San Juan Bosco, 15, planta 6. ${ }^{\text {a }}$ Oftalmología)

50009 Zaragoza

España

E-mail: jmateo1999@yahoo.es
} 
hematológica, como a los efectos secundarios del tratamiento de la misma. El nervio óptico llega a estar afectado hasta en un 13-18\% de los pacientes con leucemia $(4,5)$.

La recidiva tras una remisión completa empeora mucho el pronóstico, presentando una alta mortalidad (1).

\section{CASO CLÍNICO}

Un varón de 18 años con antecedentes personales de LAL - T en remisión completa durante 6 meses tras tratamiento quimioterápico según protocolo PETHEMA LAL - alto riesgo/2003 (Vincristina, daunorrubicina, prednisona, L-asparraginasa, ciclofosfamida, con quimioterapia intratecal) y en lista de espera para trasplante alogénico de progenitores hematopoyéticos, acudió a urgencias de nuestro hospital por presentar una pérdida de agudeza visual (AV) en ambos ojos: AV de 1/10 con ojo derecho (OD) y cuenta dedos con ojo izquierdo (OI) de dos días de evolución. Al explorar el fondo de ojo (FO), se apreció un masivo edema de disco bilateral (figs. 1 y 2). El resto de la exploración oftalmológica fue normal.

Aunque en el diagnóstico diferencial se incluían otras patologías como hipertensión arterial, diabetes, masa intracraneal, hipertensión intracraneal benigna, neuritis óptica bilateral o sarcoidosis entre otros (2), debido a la edad del paciente y, principalmente, a sus antecedentes patológicos personales, la

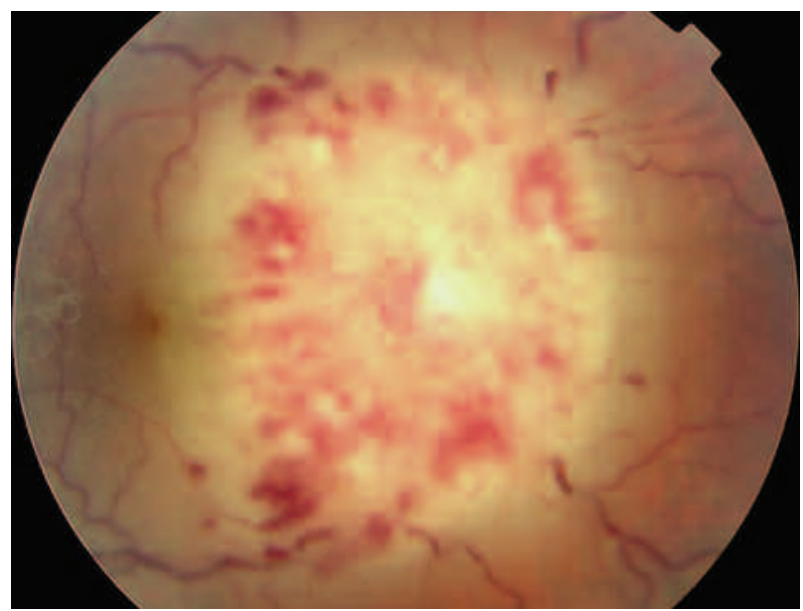

primera sospecha fue, desde el inicio, la de infiltración por LAL-T.

El resto de exploraciones resultaron normales: resonancia nuclear magnética (RNM) normal, serologías víricas negativas, punción lumbar negativa, ausencia de recaída en el hemograma.

Pese a no constatarse infiltración neuromeníngea, se administraron dosis adicionales de quimioterapia (QT) intratecal. El aspecto del FO mejoró, presentando un edema de disco óptico menos acusado (figs. 3 y 4). Durante el ingreso, el paciente sufrió numerosas complicaciones, como bronconeumonía, síndrome de distress respiratorio del adulto, hematoma sofocante de cuello y pseudotumor cerebri, y hubo que realizarle una traqueostomía.

A las seis semanas del alta, se realizó una nueva exploración de FO al empeorar la clínica visual del paciente, observándose desprendimiento de retina exudativo en OD con edema de disco bilateral (figs. 5 y 6). En ese momento, el hemograma mostró leucocitosis periférica con blastosis, y el aspirado medular una infiltración masiva LAL-T.

El paciente recibió tratamiento corticoideo y QT (fase B del protocolo Hyper CVAD y terapia intratecal), presentando sucesivas ganancias y pérdidas de AV. No se le administró radioterapia $(2,4)$, ya que la misma no está incluida en el protocolo de actuación utilizado por nuestro hospital para el tratamiento de las recaídas de LAL-T.

A pesar del tratamiento quimioterápico, no se logró una nueva remisión de la enfermedad, y el paciente falleció a los pocos meses.

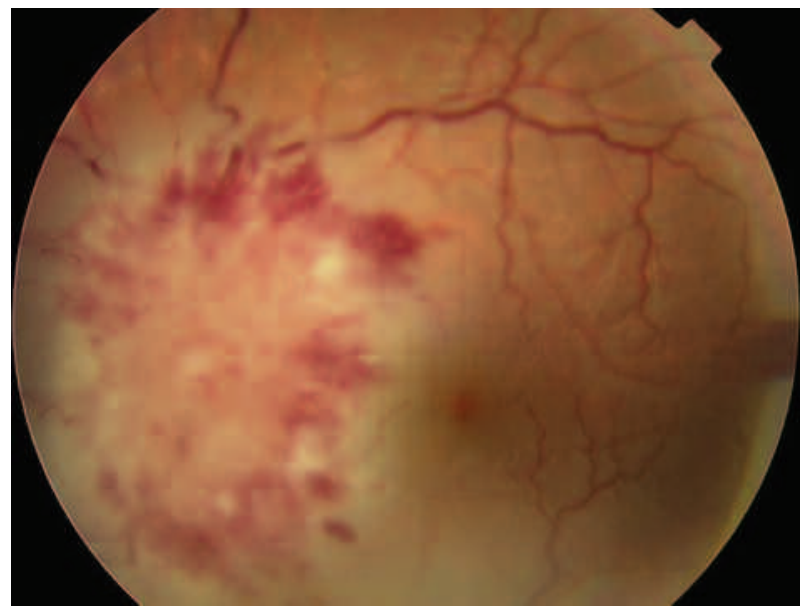

Fig. 1 y 2: Edema de disco óptico bilateral, sugestivo de infiltración por LAL-T. Gran edema de disco, con abundantes hemorragias, exudados y tortuosidad vascular. 

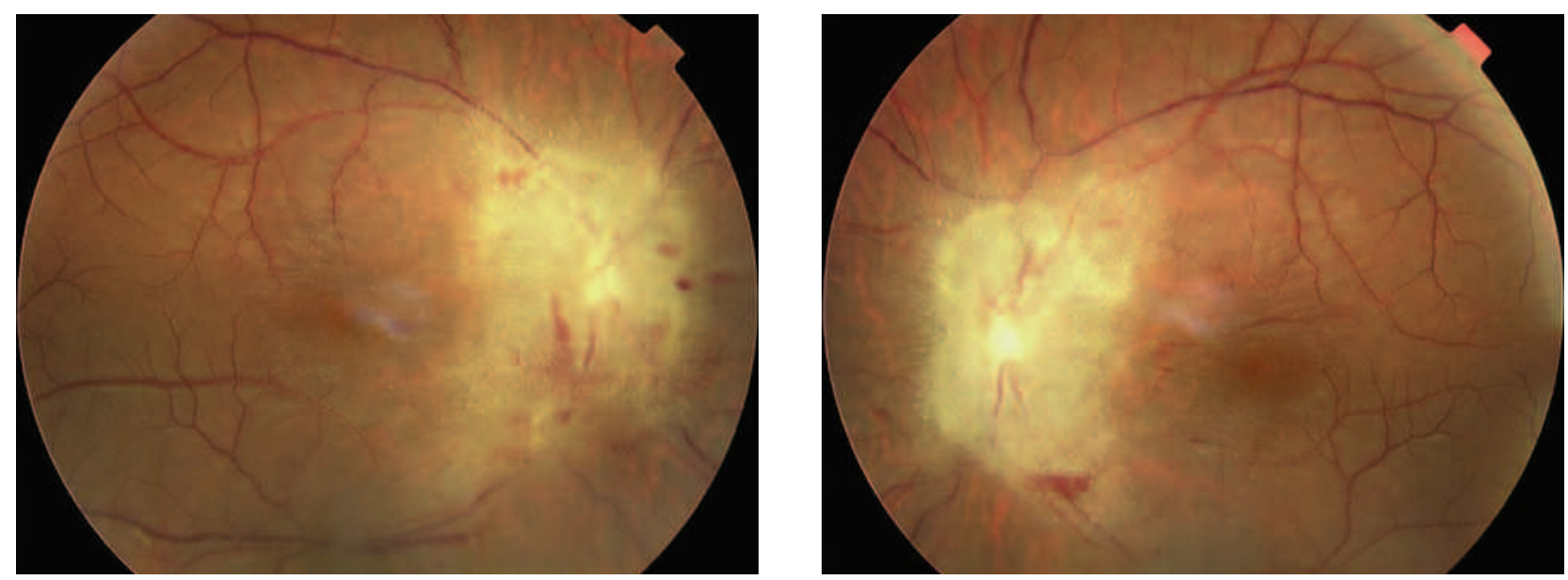

Fig. 3 y 4: Aspecto de las papilas tras la administración de las dosis extras de QT intratecal. El edema de disco óptico ha disminuido en ambos ojos.
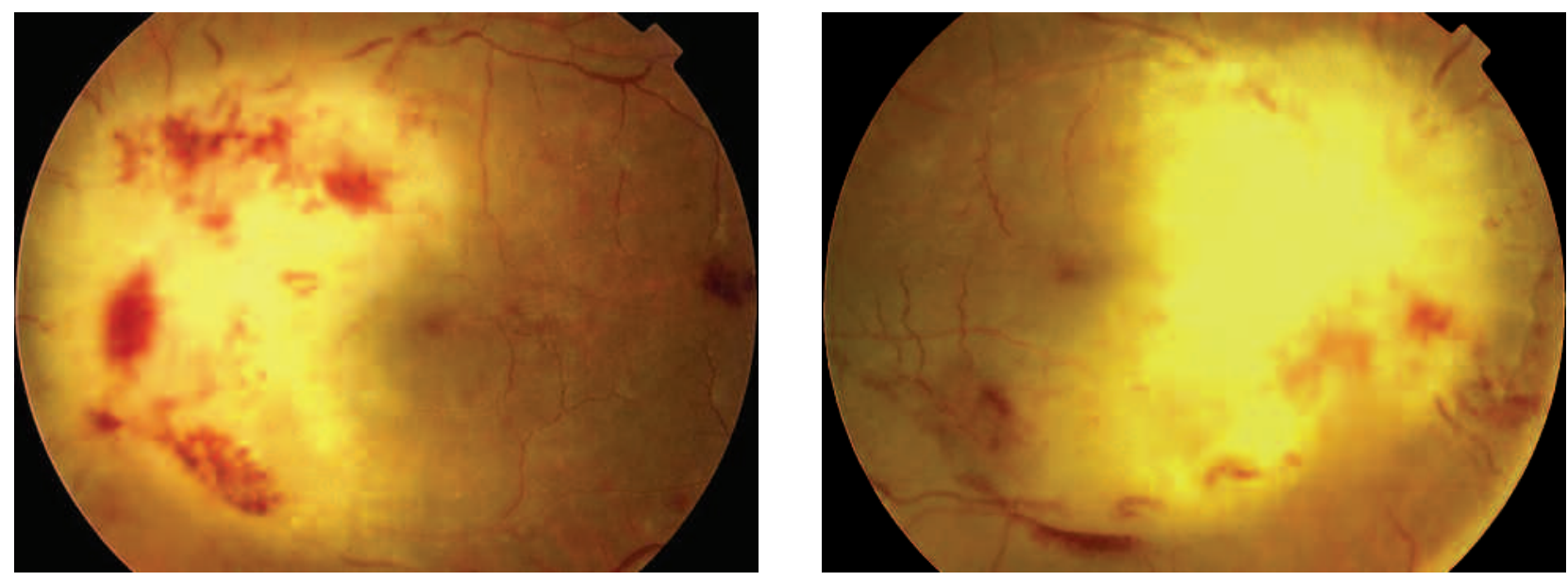

Fig. 5 y 6: Aumento del edema de disco óptico y presencia de abundantes hemorragias peripapilares, exudados y un desprendimiento de retina exudativo en $O D$.

\section{DISCUSIÓN}

La infiltración del nervio óptico puede aparecer como un signo aislado de recaída extramedular de LAL-T, precediendo incluso en varios meses a la recaída hematológica. Aunque el FO suele aparecer alterado, puede haber afectación del nervio óptico, con pérdida de $\mathrm{AV}$, pero con $\mathrm{FO}, \mathrm{RNM}$ y punción lumbar normales (4).

Es importante realizar controles periódicos en estos pacientes, incluyendo una exploración oftalmológica completa, con fondo de ojo, especialmente si presentan clínica visual, ya que una detección más temprana de la recidiva, con una instauración precoz del tratamiento, puede mejorar su pronóstico vital. El pronóstico visual siempre es muy pobre $(3,4)$.

\section{BIBLIOGRAFÍA}

1. Ortega JJ. Leucemias agudas infantiles. En: Sans-Sabafren J, Besses Raebel C, Vives Corrons JL. Hematología Clínica 5. ${ }^{a}$ ed. Elsevier 2006; 437-450.

2. Mayo GL, Carter JE, McKinnon SJ. Bilateral optic disk edema and blindness as initial presentation of acute lymphocytic leukemia. Am J Ophtalmol 2002; 134: 141 142. 
3. Nikaido H, Mishima H, Ono H, Choshi K, Dohy H. Leukemic involvement of the optic nerve. Am J Ophtalmol 1988; 105: 294-298.

4. Schocket LS, Massaro-Giordano M, Volpe NJ, Galetta SL.
Bilateral optic nerve infiltration in central nervous system leukemia. Am J Ophtalmol 2003; 135: 94-96.

5. Arruga J. Lesiones metastásicas y paraneoplásicas del nervio óptico. Rev Neurol 2000; 31: 1256-1258. 